\title{
RESIDENTIAL MOBILITY AND URBAN-RURAL RESIDENCE WITHIN LIFE STAGES RELATED TO HEALTH RISK AND CHRONIC DISEASE IN TECUMSEH, MICHIGAN
}

\author{
Helen low Metzner, ERnest Harburg and Donald E. Lamphiear \\ University of Michigan School of Public Health, Ann Arbor, U.S.A.
}

(Received in revised form 19 April 1981)

\begin{abstract}
Residential history, diagnosis of three chronic diseases, five clinical measurements and histories of smoking and drinking were obtained from a sample of 615 women and 529 men, aged 35-69, randomly selected from respondents of the Tecumseh Community Health Study. Two measures of residential mobility and one of urban-rural residence, during early life stages and over the entire lifetime, were related to subsequent adult health traits. Greater residential mobility, particularly in childhood but also in later life, was significantly associated with greater prevalence of hypertension and higher mean diastolic blood pressure in older persons. Greater duration of urban residence was associated with greater prevalence of chronic bronchitis. Both residential traits were associated with greater prevalence of CHD, and with behavior patterns, namely cigarette smoking and drinking, that are risk factors for certain chronic diseases.
\end{abstract}

A GENERAL awareness by professionals and laymen of the association between states of health and disease and usual frequent and infrequent life events has generated increased activity in recent decades seeking to substantiate these observations by systematic scientific research. Most frequently studies have related selected acute or chronic diseases, morbidity in general and mortality to particular life events or an index of life events experienced in some recent defined interval. These studies have had mixed success thereby stimulating further research in the quest for better definitions of the events, their stressful attributes and their systematic associations with specific health states. The following report is from one of a series of such studies.

This research is a continuation of the longitudinal and cross-sectional analyses of the relationships between the psychosocial aspects of ordinary life experiences and health status of participants in the Tecumseh Community Health Study. In an earlier paper [1], childhood experiences of social incongruities or discontinuities, as expressed by residential mobility, parental deprivation and parental status incongruities occurring 30-60 yr ago, were related to current adult health status variables comprised of selected health risk factors and prevalence of certain chronic diseases. Among these experiences, only childhood residential mobility was found to be significantly associated with certain health variables and only consistently among women members of the adult population studied.

In the present report, the analyses were carried further to assess whether inconguities experienced at a later period in life and cumulatively over the life span were associated with adult health status. The incongruities were represented by two kinds of measures, residential mobility in time periods subsequent to the earliest and added to the earliest for total cumulative experience and secondly, by proportion residential time in urban or rural environments at various periods of life and cumulatively over the total life span. Answers to three questions were sought. First, was mobility in the early adult period and 
over the life span related to later health? Second, were the effects of mobility stronger in one age period and additive or interactive across periods? Third, was a greater degree of urban residence, with its potential for more social incongruities and stress than rural residence, associated with poorer health status and was moving between rural and urban similarly detrimental?

\section{METHOD}

Data for this analysis conte from two phases of the Tecumseh Community Ilealth Study (TCHS), a longitudinal epidemiological study of selected acute and chronic diseases in a whole community in Michigan. The study's field operations began in 1957 and are still continuing. At the time of the data collections for this study, the focus was primarily on ascertaining the precursors of coronary heart disease (CHD). hypertension, diabetes, chronic bronchitis, asthma and hay fever. Objectives and methods of the overall study have been described by Epstein et al. [2] and Napier et al. [3].

\section{Sample}

The sample for the present study consists of a subset of the whole Tecumseh Study population who participated in the second series of examinations in 1962-1965. The participation rate for that series was $85 \%$. The subset, more fully described earlier [1], is made up of 529 men and 615 women, 35-69 yr of age, interviewed in 1966-1967, who were living in a $40 \%$ stratified area sample of dwelling units in the study area.

\section{Measures}

Residence history, family composition, and occupation history were obtained in 1966-1967 by a $1-2$ hr personal interview directly with the respondent, conducted in the home by trained interviewers. Chronological data were reduced in coding to yield the residence and mobility measures described below. Health history data were obtained in 1962-1965 by a $1 \mathrm{hr}$ direct personal health interview conducted in the home. Other health data were gathered at that time during a $1 \mathrm{hr}$ clinic visit from tests administered by technicians and examinations conducted by physicians.

Residential status and mobility were determined for broad age periods defined by stages of social development. Periods of interest are: childhood, through age 16; young adulthood, ages 17 through $29 \mathrm{yr}$; these two periods combined: and total lifetime up to age at interview. The developmental periods are obviously not independent, but variables are defined within the age ranges (periods) to describe different psychosocial developmental contexts of residential experience. Residential variables for childhood and young adulthood are defined independently. The respondent is classified as high or low mobility for the period, within the cohort defined by sex and current age (middle or older), according to total number of moves and as having primarily rural or urban residence according to number of years in places of size 2500 or less. In defining the combined period (aged 0-29), the respondent is classified as 'low' for the mobility variables and 'rural' for residential status if he or she falls in those categories in both childhood and early adulthood, and as 'high mobility' or 'urban' for the combined period if he or she falls in those categories in one or both of the component periods. Thus, the variable indicates whether or not there is continuity of the experience of low mobility or rural residence over the longer period, and the importance of the component periods is retained in the definition. Over the entire lifetime, which includes the years in earlier periods and others subsequent, definition is again based on thresholds but without regard to period of occurrence; the variables simply measure total exposure up to the time of interview.

Results are reported for males and females separately in two ranges of current age, 35-49 yr (middle) and 50-69 yr (older) in order to identify whatever sex and cohort differences occur. Cohort differences that occur are significant in that they may be related to physiological or social aging, historic factors characteristic of the cohort's childhood 
or other periods as World War I, II, the Great Depression, etc. all of these and interactions among them and the health variables.

Three aspects of residence are discussed here. Two are direct measures of change in residence: number of dwelling units lived in and numbers of places lived in. These variables were determined by asking the respondent each place lived in from birth to the present, and at how many addresses in each place. Definitions of residential mobility variables for the age periods of concern are given in Table 1.

The third variable, urban-rural character of residence, was evolved during reconsideration of the size of place variable used in the earlier study [1]. Analysis of the association between six size classes and the health variables showed an urban-rural effect with the population threshold at 2500 . The urban-rural residence is defined, accordingly, in terms of the number of years in the period of interest during which the respondent lived in places with a population of 2500 or fewer (see Table 2).

Health traits are the presence or absence of chronic disease and the values of health risk variables. The diagnosed or defined chronic conditions are (1) chronic heart disease, (2) hypertension, and (3) chronic bronchitis. The health risk factors are (1) whether the respondent ever smoked ('ever' includes current and ex-smokers), (2) whether the respondent ever drank ('ever' includes current and ex-drinkers), (3) number of cigarettes smoked per day, (4) amount of alcohol consumed per week, in units equivalent to 5 grams of

TABLE 1. NUMBERS AND PERCENTAGES OF RESPONDENTS IN RESIDENTIAL MOBILITY CATEGORIES IN FOUR AGE INTERVALS, BY SEX AND CURRENT AGE

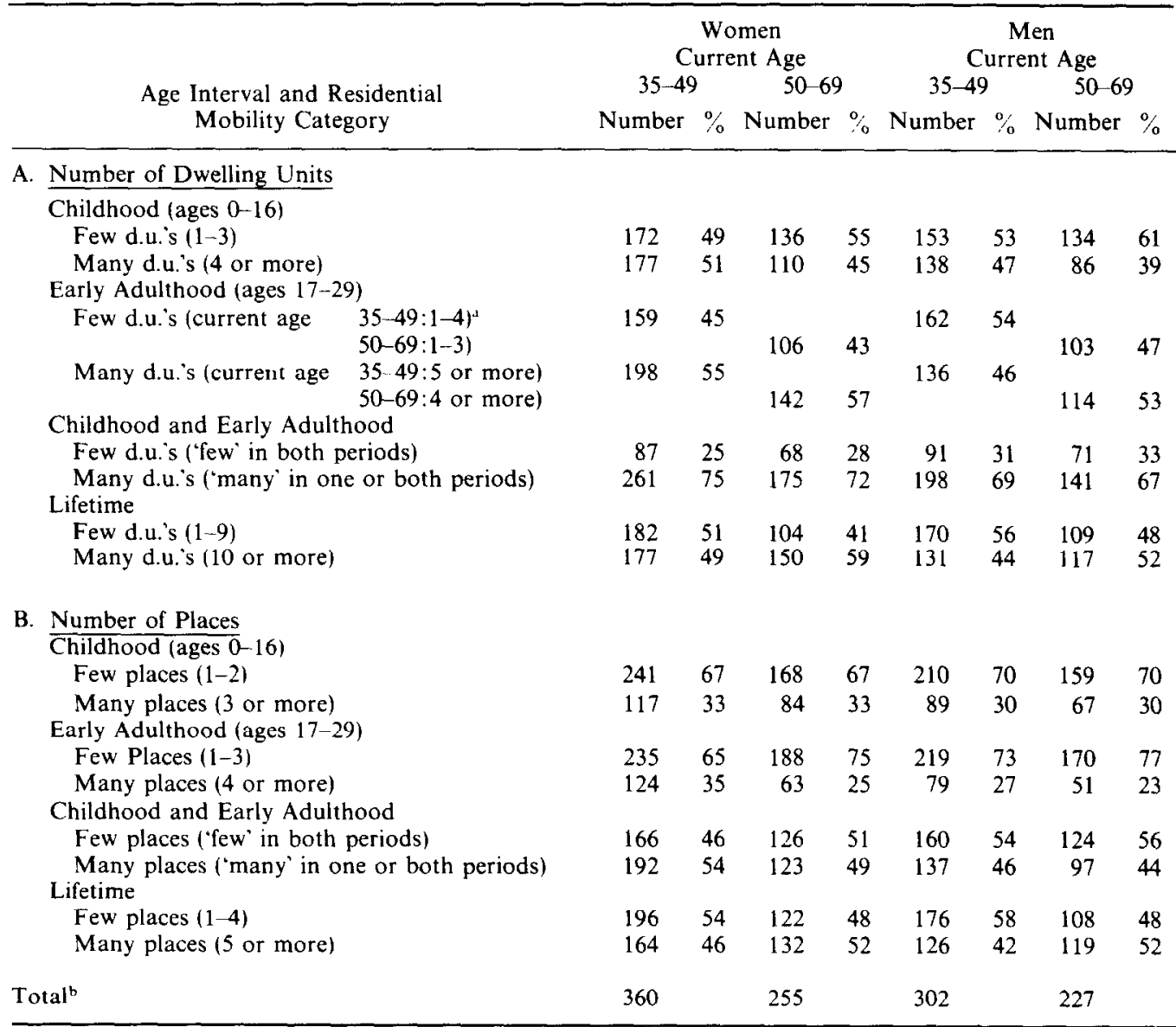

${ }^{\top}$ Number of dwelling units was partitioned near the median into few and many according to the distributions of each current age group.

${ }^{b}$ Differences between totals for the sex-age groups and sums of the $N$ 's in each partition are due to missing information for mobility. 
TABLE 2. NUMBERS AND PERCENTAGES OF RESPONDENTS IN URBAN-RURAL CATEGORIES IN FOUR AGE INTER VALS, BY SEX AND CURRENT AGE

\begin{tabular}{|c|c|c|c|c|c|c|c|c|c|}
\hline Age interval and amount of & ne lived & & $\begin{array}{l}\text { Wo } \\
\text { urre }\end{array}$ & $\begin{array}{l}\text { men } \\
\text { nt age }\end{array}$ & & & $\begin{array}{r}M \\
\text { urre }\end{array}$ & len & \\
\hline in rural places (Population: 25 & or fewer) & $35-49$ & & $50-69$ & & $35-49$ & & $50-69$ & \\
\hline Definition & Classification & Number & $\%$ & Number & $\%$ & Number & $\because$ & Number & $\%$ \\
\hline Childhood (ages 0-16) & & & & & & & & & \\
\hline Few years $(0-15)^{a}$ & Urban & 186 & 52 & 98 & 38 & 157 & 52 & 81 & 36 \\
\hline Many years $(16-17)$ & Rural & 174 & 48 & 157 & 62 & 144 & 48 & 146 & 64 \\
\hline Early adulthood (ages 17-29) & & & & & & & & & \\
\hline Few years $(0-6)$ & Urban & 228 & 63 & 114 & 45 & 195 & 65 & 91 & 40 \\
\hline Many years $(7-13)$ & Rural & 132 & 37 & 141 & 55 & 106 & 35 & 136 & 60 \\
\hline Childhood and early adulthood & & & & & & & & & \\
\hline $\begin{array}{l}\text { Few years ('few' in one or both } \\
\text { periods) }\end{array}$ & Urban & 267 & 74 & 144 & 56 & 228 & 76 & 116 & 51 \\
\hline Many years ('many' in both & & & & & & & & & \\
\hline periods) & Rural & 93 & 26 & 111 & 44 & 73 & 24 & 111 & 49 \\
\hline Lifetime & & & & & & & & & \\
\hline Few years $\left(0-75^{\circ}{ }_{0}\right.$ of lifetime $)$ & Urban & 288 & 80 & 205 & 80 & 248 & 82 & 174 & 77 \\
\hline $\begin{array}{l}\text { Many years }\left(76^{\circ}{ }_{n} \text { or more of life- }\right. \\
\text { time) }\end{array}$ & Rural & 72 & 20 & 50 & 20 & 53 & 18 & 53 & 23 \\
\hline Total $^{\mathrm{h}}$ & & 360 & & 255 & & 302 & & 277 & \\
\hline
\end{tabular}

"Few years in rural places in childhood was defined as $0-15$ yr for two reasons: the median of the population occurs between 15 and $16 \mathrm{yr}$ and the frequency distribution is flat between 0 and $15 \mathrm{yr}$.

'Differences between total for the sex-age groups and sums of the $N$ 's in each partition are due to missing information for place of residence.

alcohol, (5) forced expiratory volume in one second, F.E.V.1.0. a measure of lung function, (6) adiposity index, (7) serum cholesterol, (8) systolic blood pressure and (9) diastolic blood pressure, fifth phase. For more detailed descriptions of these variables and their distributions in the study sample, see Metzner et al. [1].

\section{Statistical analysis}

The three categorical health status variables were dichotomized, and tests of significance were computed within age groups by Fisher's exact test. For continuous risk factors, the test of significance was the $t$-test for the difference between means.

The hypothesis tested for residential variables is that more time spent in rural environments and lower levels of mobility are associated with lower prevalence of chronic disease, lower mean levels of the physiological risk factor variables, lower levels of obesity and less frequent cigarette smoking and drinking. Formally this hypothesis is the alternative to the null hypothesis, that there are no differences in the predicted direction associated with classification by the residential variables. The test of significance appropriate to an hypothesis predicting one direction is a one-tailed test.

\section{RESULTS}

Ordinarily once the number of comparisons has been set by the design of the study, the expected number of significant differences under the null hypothesis is fixed by the significance level. However, the confidence interval about the expected number cannot be calculated in the present study because of the logical dependence in the definitions of certain sets of variables. There is, however, a large excess of statistically significant differences, in the direction predicted by hypothesis, falling into definite patterns.

\section{Number of dwelling units (Tables 3 and 4)}

There are more significant associations between dwelling unit mobility and health among women than among men. Dwelling unit mobility was related most consistently to 


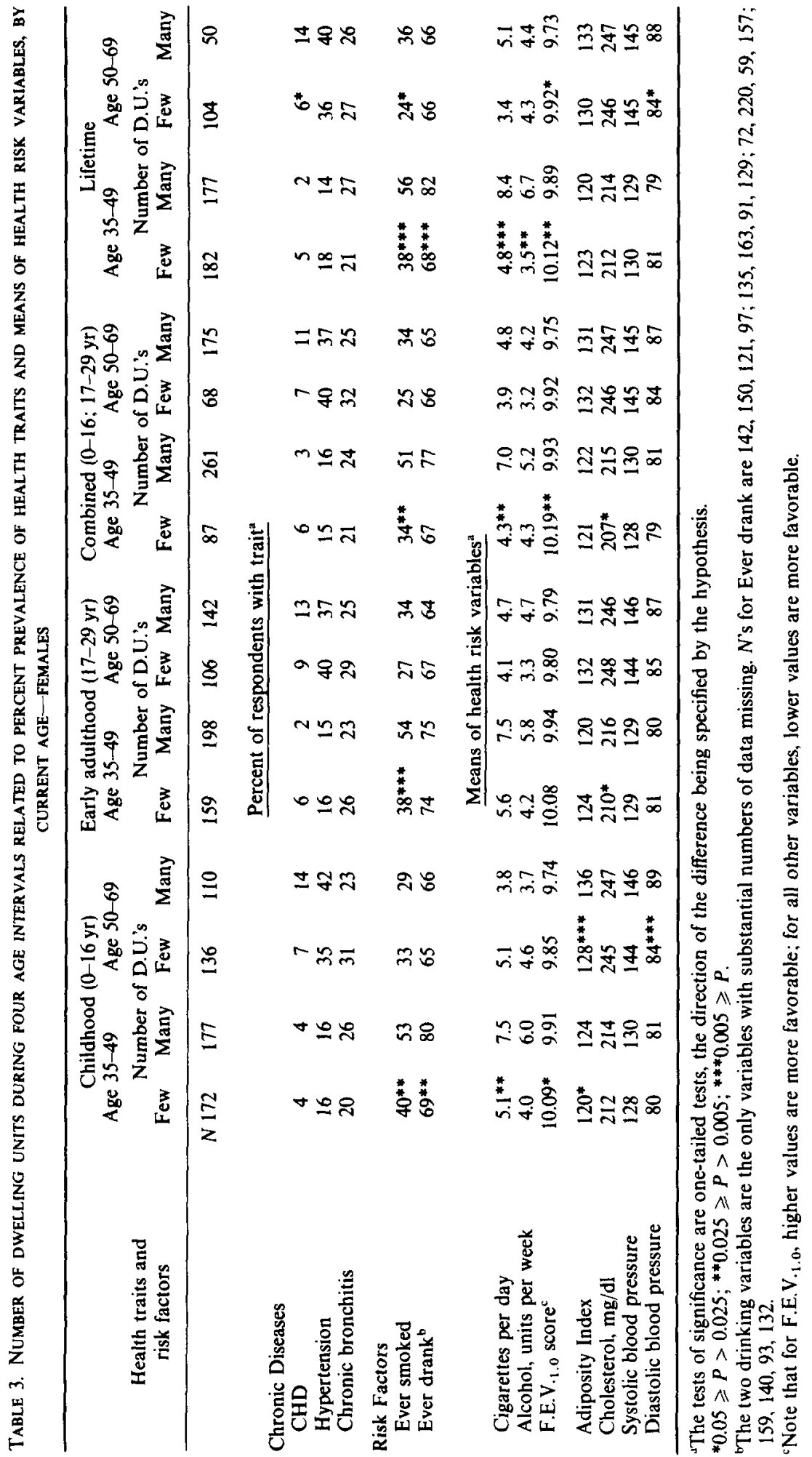




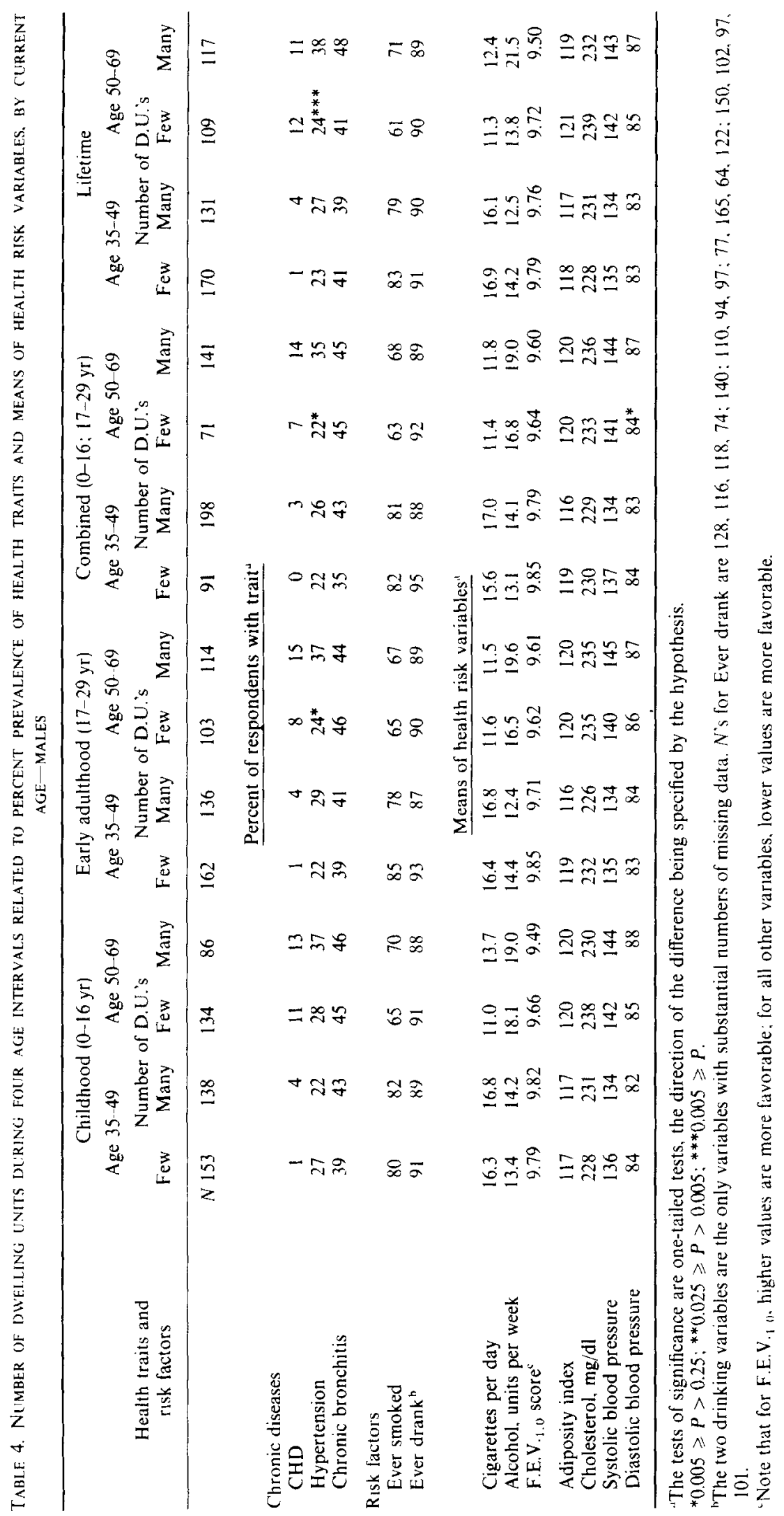


cigarette smoking, drinking and F.E.V.1.0 score in middle age women and hypertension in older men and higher diastolic blood pressure in both sexes in the older group.

In women, considering the four developmental periods studied, cumulative lifetime mobility and mobility in childhood $(0-16 \mathrm{yr})$ seemed to be more significant in relation to current health status than the other periods. More middle age women who were dwelling unit mobile during their lifetimes had ever smoked and ever drunk, currently smoked and drank more, and had poorer F.E.V. .1.0 $_{\text {o }}$ scores than their less mobile sex-age peers. Older women who were mobile over their lifetimes were more likely to have CHD and to have ever smoked. Similar associations were not found for middle age men. More older men who were dwelling unit mobile over their lifetimes and during their early adulthood had hypertension. Early childhood experiences in dwelling unit mobility were not associated significantly with men's adult health.

\section{Number of places (Tables 5 and 6 )}

There are many more significant associations among women than among men between health and mobility as measured by number of places or locations lived in.

Place mobility was related most consistently across age periods to smoking among middle age women. The place mobile middle age women smoked more cigarettes currently and had poorer F.E.V.1.0 scores. They were also more likely to have ever drunk alcoholic beverages. Older women, who were mobile in childhood and in the combined period ages $0-29$, were more likely to have CHD. Lifetime place mobility was not related to CHD for them. Among older men, place mobility in the combined period was related to hypertension and diastolic blood pressure as measured some $20-40 \mathrm{yr}$ later.

It is interesting to note that there are indications in the data counter to the hypothesis that greater mobility is associated with poor health. Men who were place mobile had smaller proportions who ever smoked and drank and they currently smoked and drank less and had lower cholesterol levels.

\section{Proportion of time in rural places (Tables 7 and 8 )}

Time spent in residence in rural areas seems to be more important than either mobility measure in relation to the health measures studied. Men and women with longer rural residence have more beneficial levels of current health in all age periods and this is especially true for women. The health factors affected are measures of smoking and drinking and factors closely related to these behaviors, namely, chronic bronchitis and less often, CHD in older women.

\section{Childhood in rural places and current CHD and bronchitis}

The relationships between time lived in rural places during childhood and chronic bronchitis in men and women and CHD in women were of sufficient interest to merit more intensive analysis. The question was whether these two conditions were related to residence in rural places independently of factors known or hypothesized to be related to both the health condition and the residence variables. Smoking habit was selected as a concomitant variable because it is known to be related positively to chronic bronchitis and $\mathrm{CHD}$, and was found to be highly related negatively to time in rural places. Father's occupation during the respondent's childhood was selected as a second concomitant variable to partition rural residence into early experience in a farm life environment and other rural environment. Contingency table analysis was employed using the MantelHaenszel chi-square statistic summarizing differences over levels of a covariable set and a standardized risk index. [4] Rural residence during childhood was still significantly negatively related to CHD among older women $(P<0.05)$. However there was sufficient inconsistent interaction among the respondents' current smoking status, their fathers' occupation when they were growing up and rural residence to cloud the meaning of the relationships between residence and CHD. Early rural residence was negatively related at a marginal level to chronic bronchitis $(P<0.10)$ only among middle age women, taking 


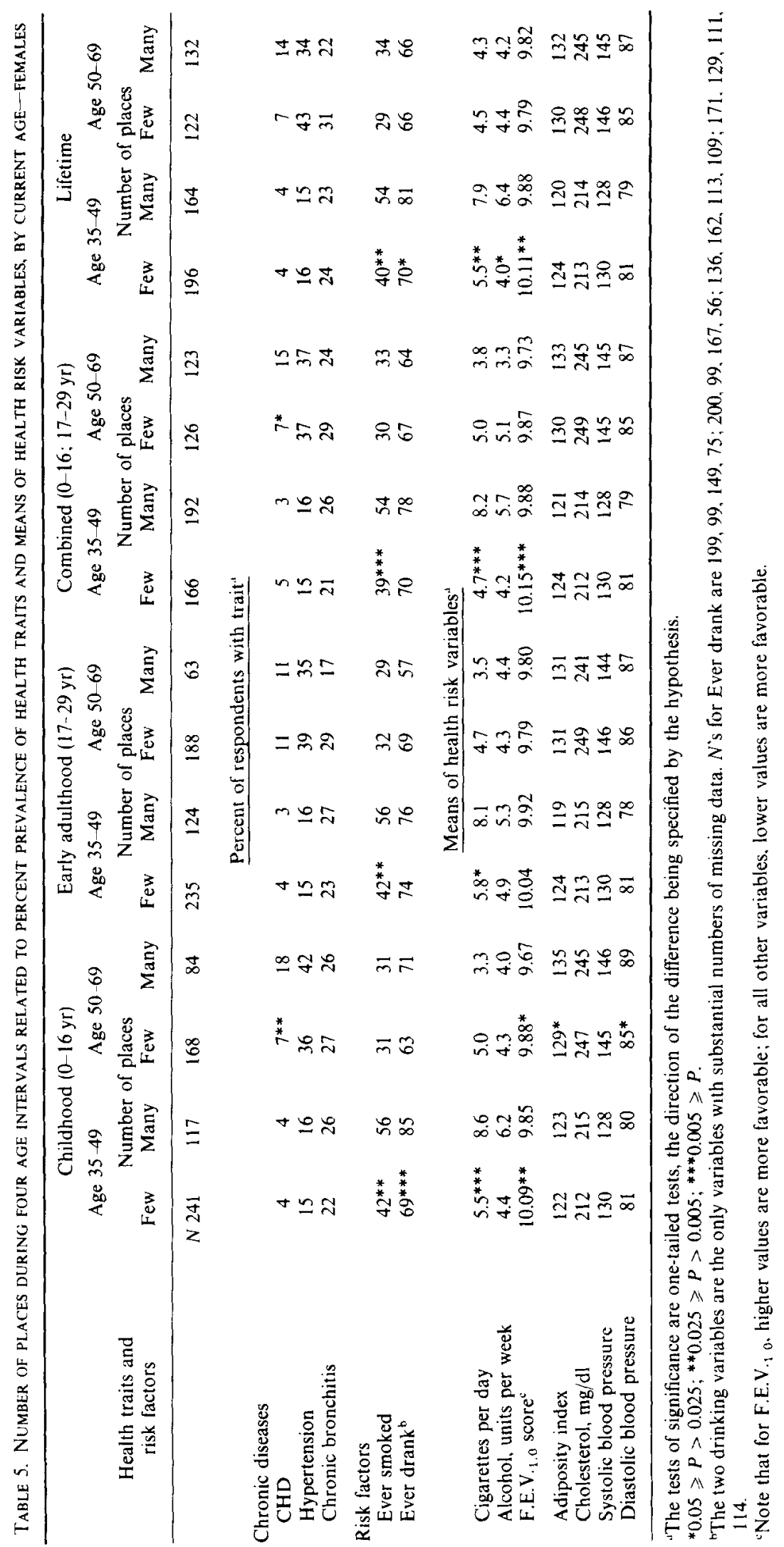




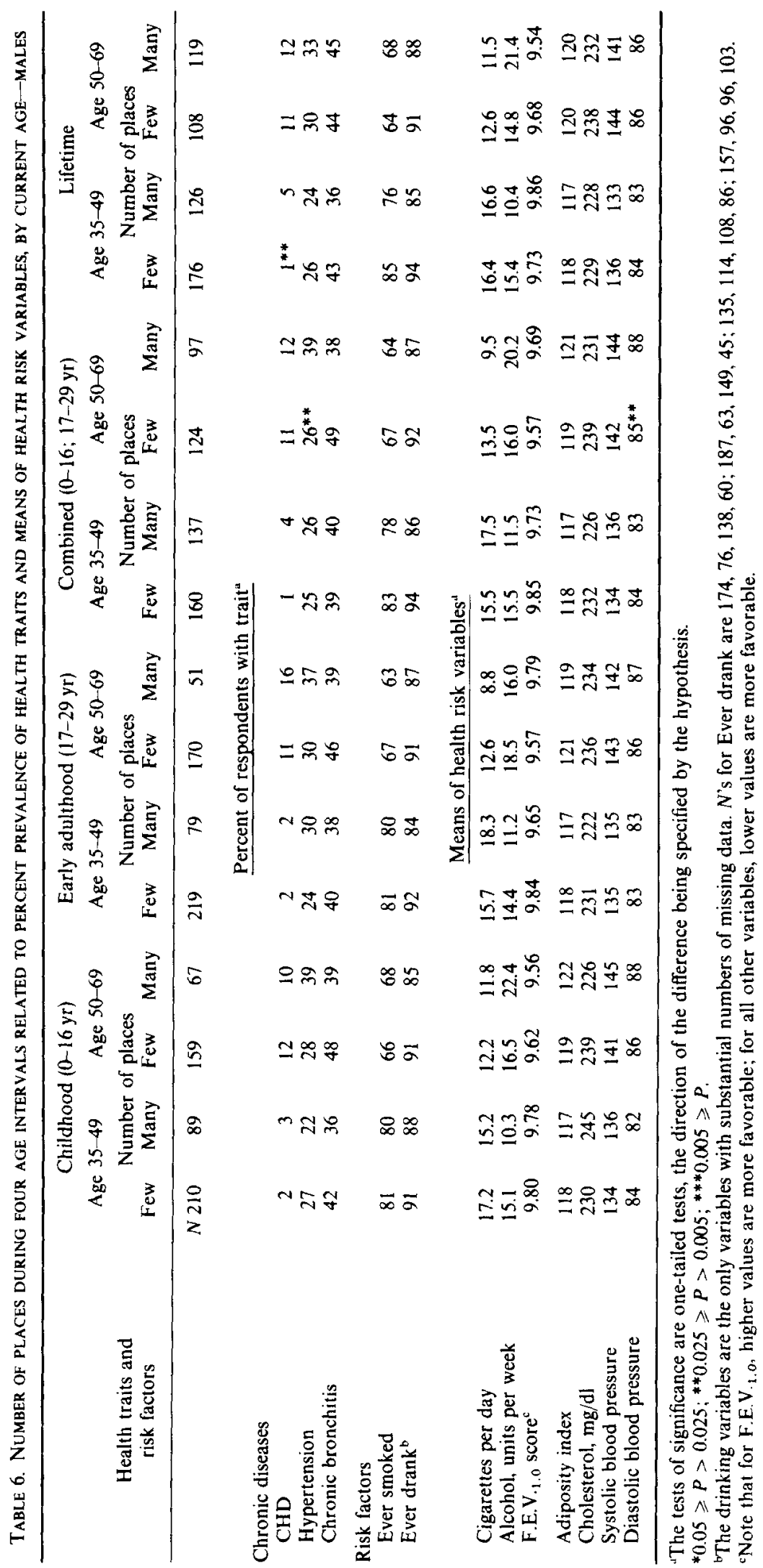




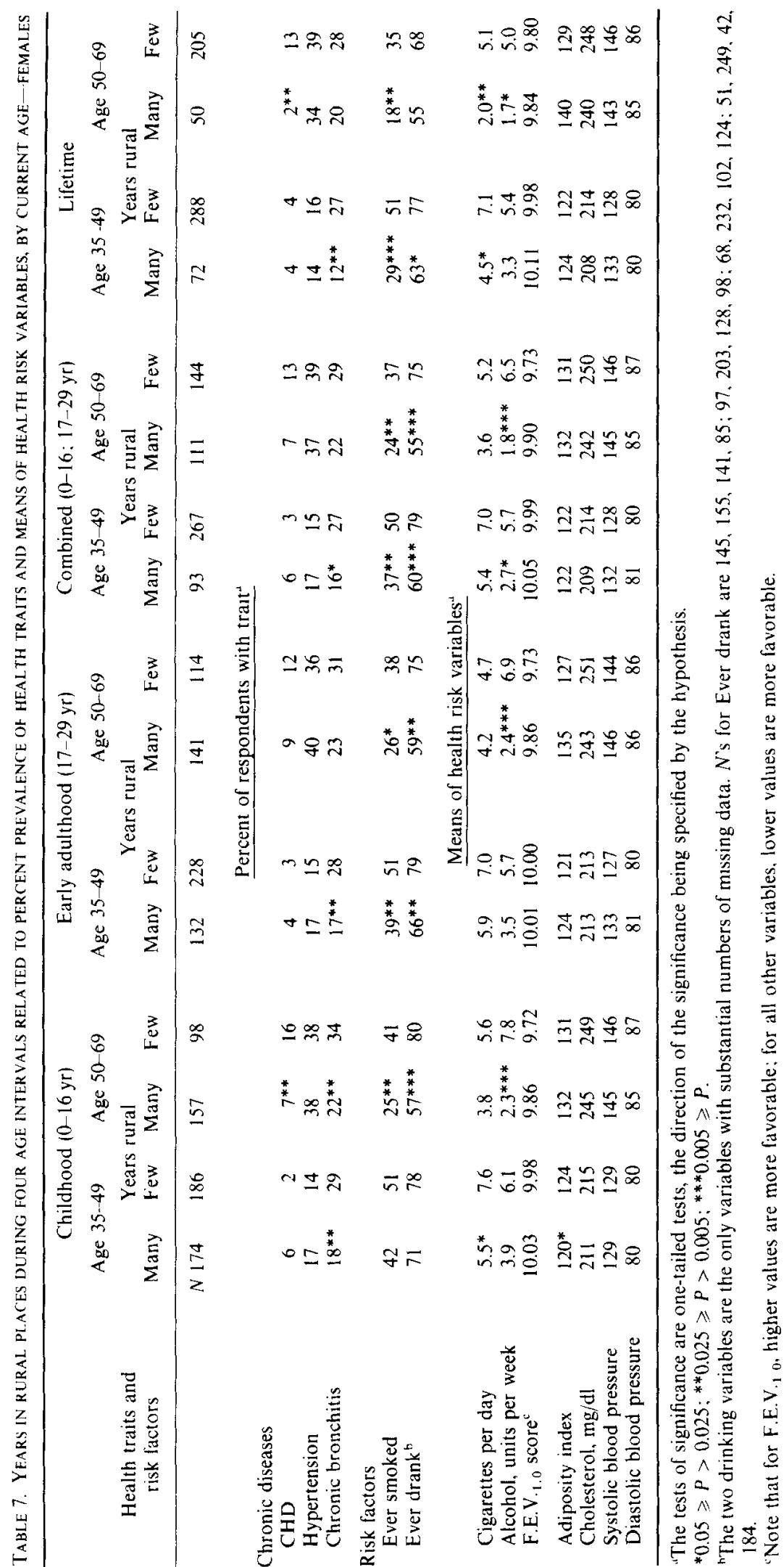


into account smoking status and fathers' occupation. There was no apparent interaction among the variables in this analysis. The standardized relative risk index for these women was 1.2 , a rather small value.

\section{DISCUSSION}

\section{Measures}

It should be noted that the 12 health traits, considered as dependent variables, are not all independent measures. Hypertension was defined as a systolic blood pressure of 160 or over, or a diastolic blood pressure of 95 or over, as found on the medical examination. Hypertension and blood pressure as a continuous measure are related. Number of cigarettes smoked currently is highly related to whether the respondent ever smoked, since non smokers' and ex-smokers' zero quantities are included in the calculation of the mean. The same relationship holds for alcohol drinking practices and quantity of alcohol consumed. Although separate items are related by definition, they differ conceptually and in the way they relate to the mobility variables. The two blood pressure measures are related physiologically, and empirically they are highly correlated. Nevertheless both are important in the etiology of chronic diseases.

The mobility measures are also related by definition. Those who lived in only one dwelling unit must have lived in only one place, and number of dwelling units cannot be smaller than number of places. Beyond these necessary relationships, number of dwelling units and number of places do not have to be closely related. Their correlation coefficient was about 0.7 for residence experiences during childhood. The urban-rural measure is not necessarily related to the mobility measures since few or many moves may be made without changing size classification. For the present sample, however, the more time spent in rural places, the fewer moves were made. Also, size of place was determined as of the time the respondent lived there; therefore it was possible for a respondent to have lived in only one place, Tecumseh for example, and to be classified as living in both rural and urban areas. Tecumseh was classed as rural, under 2500 population, up to the 1940 census and after, as urban, having grown steadily until 1960 levelling off at 7000 .

Nor are age periods independent. Childhood and early adulthood were independently ascertained, but the other two periods include these periods by definition. Measures for the cumulative periods, $0-29 \mathrm{yr}$ and lifetime experience were constructed differently for different purposes. Mobility and urban-rural residence during the combined childhood and early adult periods were defined relative to those periods and not by total number of dwelling units, places, or number of years, in order to retain the importance of those age periods in evaluating experience. Fewer dwelling units, fewer places and more years in rural places during the combined period meant that relatively fewer dwelling units or places and more years in rural places had to be experienced in both early periods, categories representing consistent low mobility or rural residence for these periods. Tendencies for relationships between urban-rural residence and mobility and health traits that might be too weak to show in either early period, were expected to be strengthened and manifested in the combined period. In the total lifetime period, experience over the entire age range was counted, regardless of current age, as a measure of total exposure to the experience.

The self reported behavioral measures were not validated. These include names of places lived and length of stay and smoking and drinking habits. Validity of self reports of mobility has not been discussed extensively in the literature, but the validity of smoking and drinking reports has. Indications are that questionnaire responses about smoking behavior are internally consistent, reliable and more valid than existing physiologic measures of smoking behavior [6]. The question of the validity of reports of drinking behavior has not been adequately answered. The literature shows under-reporting by alcoholics and under-reporting when comparing extrapolated national consumption on the basis of interview data with alcohol sales figures. There has been no population study 
relating independent observations of drinking behavior with self reports. However, internal consistency in the data in this study and in others and similar relationships among drinking reports and other variables across studies give confidence that the data obtained by self report generally measure relative drinking behavior.

\section{Mobility}

Significant relationships between the two mobility variables and the health traits show four interesting patterns. The most consistent relationship is between more mobility at all age periods among middle age women and cigarette smoking - ever smoked and number of cigarettes currently smoked. F.E.V.1.0 ${ }_{\cdot 1.0}$ values for these women also are lower for the more mobile during the combined and lifetime periods. Higgins et al. [5] have already shown that cigarette smoking and F.E.V. values in the Tecumseh population are highly related. Somewhat less consistently across age periods, the more mobile middle age women were more likely to be current or past drinkers. In contrast, the data show that fewer middle age men in the Tecumseh population who were place mobile were cigarette smokers or drinkers, contrary to the findings of Lilienfeld [7], who found a tendency for men smokers to be more residence mobile.

The positive relationship of mobility with smoking and drinking for middle age women may be explained in terms of two factors, stressful circumstances and social norms. Although no one knows all the reasons why people smoke or drink nor why any one person smokes or drinks, the factors of stress and social norms are recognized as significant and frequently occurring. Residential mobility is stressful, at the very least in the short run, as all who have experienced it can attest. What is investigated here is whether there are long term effects on selected measures of health and health behavior assuming that the stressful aspects last and overcome positive aspects of mobility. Stress is related to smoking and drinking. In another analysis of Tecumseh data using a larger cohort examined at a later time, other more apparent measures of stress were positively related to cigarette smoking among men and women and drinking among men. These measures were marital status categories of separated and divorced and indexes of reported worry and effects of worry on social and work life. In our group, more of the mobile middle age women may have smoked or drunk to relieve tension or because they found these behaviors acceptable in their new environments, or both.

In the Tecumseh population it was not the norm for women to smoke; less than half the women respondents did so. However, over half the women said they drank, either currently or in the past. Although drinking to some extent seems to be done by a large proportion of women, and readily acknowledged, there is a lot of ambivalence about the appropriateness of it for women. Among those women who moved relatively often, there were more who smoked and drank, possibly because they found these behaviors the norm in the various environments thay were exposed to.

The relationship between mobility, smoking and F.E.V. was the same for older women as for middle age but appears significant only for the entire lifetime. Fewer older women ever smoked. All U.S. smoking data show a strong negative correlation between age of women and number of smokers [8].

The lack of parallel associations between mobility and smoking and drinking among men may be related to the fact that many social norms are quite different for men and women. Relationships of smoking and drinking with age, education and income are different for men and women and the meanings of these behaviors may also be different.

There are no impressive consistent relationships between CHD and dwelling unit or place mobility for older men since significant associations are sporadic. Almost all the non-significant associations, however, tend in the same direction. Among middle-age men, cumulated life experiences of place mobility were significant for the prevalence of CHD. These few significant findings suggest that mobility is relevant to the prevalence of CHD but at this analytic level, is neither strong nor explanatory. 


\section{Urban-rural}

Among urban-rural comparisons, the most consistent and striking relationships were between proportion of time in rural places in all age periods and smoking and drinking among women, smoking among older men, and to some extent, drinking among middle age men. More time spent in rural residence was associated with fewer women and older men who ever smoked, or lower mean number of cigarettes smoked, or both. The pattern was set by residence experience in childhood, maintained for early adult experiences, not augmented by the combination, but to some degree augmented by later experiences as evidenced by the proportion found in the lifetime period.

In older women drinking followed the same pattern as smoking. In both middle age men and women, rural residence in childhood showed no association, but urban residence in early adulthood showed a stronger association with current drinking which was maintained in the later periods of urban residence. The same relationship between smoking and current residence in rural areas was found by Haenszel et al. [9] in their own study and others they reviewed. Also Higgins et al. [10] found parallel smoking differences in the larger body of the Tecumseh population according to whether current residence was within the city limits (population 7000) or outside, which included suburban and farm residences. Similar urban-rural differences in current alcohol drinking were found in a national population study by Cahalan et al. [11].

The association between rural residence and fewer smokers and drinkers might be explained in the same terms of stress and norms as for residential mobility. Also the tighter social structure and closer social controls found to be more characteristic of rural communities reinforce these associations. As Table 8 indicates, rural residence was not associated with smoking among middle age men. Presumably rural norms for middle age male smokers did not differ from urban norms. However. the proportions of smokers among the older men and middle age and older women differed by urban-rural residence and as expected, the largest proportion of rural smokers was among the older men, the next largest among the younger women and the smallest among the older women. In this population, drinking is much more accepted than smoking. Much larger proportions of men and women have drunk alcohol to some extent and amount of time in rural residence has a less uniform effect.

Differences in prevalence of chronic bronchitis reflected the smoking relationship, particularly number of cigarettes currently smoked. Association with chronic bronchitis and cigarette smoking was found by Higgins et al. [5] in the larger population from which this study group was drawn and by others in other populations [12]. Intensive analysis of the data show that the association of chronic bronchitis with less rural residence in all but middle age women is mostly accounted for by the relationship of more smoking with more urban residence.

Most interesting is the association between rural residence in childhood for older women and men and CHD prevalence. As predicted, fewer older women with a rural childhood had CHD. However, among older men the association appears to be reversed. The same relationship was found for women additionally in the lifetime period. Others have reported that men who were living in rural areas had less CHD prevalence than their counterparts in urban areas $[13,14,15]$ and less CHD mortality [16]. Syme and his co-authors explained these findings in terms of a 'discontinuity' between life situations that occurs when moves are made from a childhood rural or non-industrialized environment to an adult urban or industrialized environment. When all those with CHD in this study were scrutinized for a pattern of shifts from rural to urban from one period to the next, no such pattern appeared.

Rural residence was found by others to be associated with lower overall mortality [17], and with fewer hypertensives [18], and in other cultures, with lower blood pressures $[14,18]$. Hypertension in this population was not related to rural residence, but in older men and middle age women, more rural residence in early adulthood was associated with higher average systolic blood pressure later. Middle age women show this same 
relationship for the combined and lifetime periods. Tyroler [19] mentions a number of studies showing a similar relationship between rural residence and higher blood pressure, principally in comparisons of rural and urban blacks living in apparently economically deprived areas. Explanations for these findings were geared to poor social and economic rural conditions and do not fit the situation in Tecumseh.

The association between rural residence in childhood and later prevalence of CHD, independent of smoking habit and father's occupation, is only suggestive etiologically, considering the different directionality for men and women and the presence of interactions.

The finding that certain kinds of childhood experiences in this population appeared significant for development of CHD and chronic bronchitis in a number of ways is congruent with the report by Yano et al. [20] that other kinds of childhood experiences in Japanese-Americans were similarly related to development of CHD. In that study, early experiences with Japanese culture were associated with lower CHD prevalence; in the Tecumseh study, early rural residence and mobility were important. Early rural residence was significant among women independent of smoking and both mobility and rural residence were related through their associations with smoking and drinking habits. Inasmuch as smoking and drinking are so highly related to many other chronic conditions, these early life experiences are likely to be significant for later health in many manifestations not included in this study.

In summary, the results of this study show that residential mobility and urban residence, particularly in childhood, are related to prevalence of adult CHD, hypertension and chronic bronchitis. They are also related to behavior patterns, drinking and smoking, that are risk factors for certain chronic diseases. Although the proportions of people in the contrasting classifications illustrating the relationships are not extremely different, and some of the relationships barely reach statistical significance, the indications are that these experiences are associated with health traits. Further investigation is warranted, particularly of interaction among psychosocial factors and risk factors, geared toward more basic and fuller explanations and preventive action early in life.

Acknowledgements-This research was supported in part by Grant GM 23954 from the National Institute of General Medical Sciences, the Center for Research in Diseases of the Heart, Circulation and Related Disorders under Program Project Grant HL 09814 from the National Heart and Lung Institute, and Grant MH 25280 from the National Institute of Mental Health. The authors wish to thank Michael Siladi for his considerable conscientious assistance in data analysis and J. Richard Landis for statistical consultation.

\section{REFERENCES}

1. Metzner HL, Harburg E, Lamphiear DE: Early life social incongruities, health risk factors and chronic disease. J Chron Dis 30: 225-245, 1977

2. Epstein FH, Napier JA. Block WD, et al. The Tecumseh Study: design progress and perspectives. Arch Env HIth 21: 402-407, 1970

3. Napier JA, Johnson BC, Epstein FH: The Tecumseh Community Health Study. In Casebook of Community Studies. Kessler II. Levin ML (Eds), pp. 25-46. Baltimore: Johns Hopkins Press. 1970

4. Mantel N, Haenszel W: Statistical aspects of the analysis of data from retrospective studies of disease. J Natn Cancer Inst 22: 719-748, 1959

5. Higgins MW, Keller JB, Metzner HL: Smoking, socioeconomic sta.us and chronic respiratory disease. Am Rev Respir Dis 116: 403-410, 1977

6. Petitti DB, Friedman GD, Kahn W: Accuracy of information on smoking habits provided on selfadministered research questionnaires. Am J Public Health 71: 308-311, 1981

7. Lilienfeld AM: Emotional and other selected characteristics of cigarette smokers and nonsmokers as related to epidemiological studies of lung cancer and other diseases. J Natn Cancer Inst 22: 259-282, 1959

8. Center for Disease Control, National Institutes of Health: Adult Use of Tobacco, 1975. (DHEW Pub. No. HRA 77-1232) Washington, D.C.: U.S. Government Printing Office, 1976

9. Haenszel W, Shimkin MB, Miller HP: Tobacco smoking patterns in the United States. Public Health Monograph No. 45 Public Health Service, U.S. Dept. Health, Education and Welfare, 1956

10. Higgins MW, Kjelsberg M. Metzner HL: Characteristics of smokers and non-smokers in Tecumseh, Michigan. I. The distribution of smoking habits in persons and families and their relationship to social characteristics. Am J Epid 86: 45-49, 1967

11. Cahalan D, Cisin IH, Crossley HM: American Drinking Practices, a National Study of Drinking Behavior and Attitudes. New Haven, Conn.: New Brunswick, N.J. College and University Press, 1969 
12. Center for Disease Control, U.S. Dept. HEW, Public Health Service: Highlights of the Surgeon General's Report on Smoking and Health. Morbidity and Mortality Weekly Report. (HEW Pub. No. (CDC)79-8017) Jan. 12, 1979, 23(1): 1-11, 1979

13. Kozarević D, Pirc B, Račić Z, et al.: The Yugoslavia Cardiovascular Disease Study. II. Factors in the incidence of coronary heart disease. Am J Epid 104: 133-140, 1976

14. Garcia-Palmieri MR, Costas R Jr, Cruz-Vidal M, et al.: Urban-rural differences in coronary heart disease in a low incidence area. The Puerto Rico Heart Study. Am J Epid 107: 206-215, 1978

15. Syme SL, Borhani NO, Buechley RW: Cultural mobility and coronary heart disease in an urban area. Am J Epid 82: 344-346, 1966

16. Tyroler HA, Cassel J: Health consequences of culture change. II. The effect of urbanization on coronary heart mortality in rural residences. J Chron Dis 17: 167-177, 1964

17. Kjelsberg M, Stamler J: Epidemiologic studies on cardiovascular-renal diseases. II. Analysis of mortality by age-race-sex-place of residence, including urban-rural comparisons. J Chron Dis 12: 456-563, 1960

18. Nadim A, Amini $\mathbf{H}$, Malek-Afzali $\mathbf{H}$ : Blood pressure and rural-urban migration in Iran. Int $\mathbf{J}$ Epid 7 : $131-138,1978$

19. Tyoler HA: The Detroit Project studies of blood pressure. A prologue and review of related studies and epidemiological issues. J Chron Dis 30: 613-624, 1977

20. Yano K, Blackwelder WC, Kagan A, et al.: Childhood cultural experience and the incidence of coronary heart disease in Hawaii Japanese men. Am J Epid 109: 440-450, 1979 